Bio - grafía. Escritos sobre la Biología y su Enseñanza. ISSN 2027-1034

Edición Extraordinaria. p.p. 1185 - 1195

Memorias del IX Encuentro Nacional de Experiencias en Enseñanza de la Biología y la

Educación Ambiental. IV Congreso Nacional de Investigación en Enseñanza de la Biología.

\title{
ESTRATEGIAS DE ENSEÑANZA HACIA LA COMPRENSIÓN DE TEXTOS CIENTÍFICOS EN EL GRADO 4 DE PRIMARIA DE LA I. E. ANAURIO MANJARREZ DE LA JAGUA DEL PILAR - LAGUAJIRA
}

\section{MANUEL DAVID GÓMEZ WILCHES CARLOS HUMBERTO BARRETO TOVAR}

\section{RESUMEN}

Desde el área de Ciencias Naturales una de las necesidades primordiales de nuestros educandos es que se apropien de las competencias científicas, pero debemos generar mayor compromiso crítico, aplicando estrategias más llamativas para combatir la dificultad en los procesos de lectura y escritura en los textos narrativos y científicos. La caracterización de la comprensión lectora en textos científicos en estudiantes de $4^{\circ}$ grado de la Institución Educativa Anaurio Manjarrez La Jagua del Pilar- La Guajira implicó una transformación en la práctica pedagógica en el área de Ciencias Naturales y Educación Ambiental. Esto propone y a la vez reafirma que los textos narrativos juegan un papel importante en la etapa escolar, concibiéndolos necesarios en la formación lectora de los estudiantes, al igual que son propicios para el mejoramiento de la comprensión de lectura (Sáenz C, Pérez J. 2016).

Haciendo un análisis se inició la reflexión del docente desde el área de Ciencias Naturales y se buscaron estrategias para la mejora de la comprensión lectora y que promovieran la formación de competencias científicas implementando las rutinas de pensamiento, la observación directa de lo que ofrece el contexto, las sesiones de estudio semanal de problemas particulares del contexto (cambios de clima, temperatura), la creación de versos vallenatos para retener conceptos propios en Ciencias Naturales y Educación ambiental y el uso adecuado de las TIC en el aula. Luego de la implementación de estrategias se mejoró la comprensión lectora y el desarrollo del pensamiento científico permitiendo construir conceptos, modificarlos y descubrir interpretaciones desde el contexto.

PALABRAS CLAVES: Competencias científicas, desarrollo del pensamiento científico, comprensión de textos científicos, estrategias de enseñanza, TIC.

\section{ABSTRACT}

From the area of natural sciences one of the primary needs of our students is that they take over the scientific competences, but we must generate a greater critical commitment, applying strategies more striking to combat the difficulty in the processes of reading and writing in the narrative texts and Scientists. The characterization of reading comprehension in scientific texts in 4th grade students of the Educational Institution Anaurio Manjarrez La 


\title{
Bio - grafía. Escritos sobre la Biología y su Enseñanza. ISSN 2027-1034
}

\section{Edición Extraordinaria. p.p. 1185 - 1195}

\author{
Memorias del IX Encuentro Nacional de Experiencias en Enseñanza de la Biología y la \\ Educación Ambiental. IV Congreso Nacional de Investigación en Enseñanza de la \\ Biología.
}

Jagua del Pilar-La Guajira involved a transformation in pedagogical practice in the area of Natural Sciences and Environmental Education. This proposes and at the same time reaffirms that narrative texts play an important role in the school stage, conceiving them necessary in the reading training of students, just as they are conducive to improving reading comprehension (Sáenz C, Pérez J. 2016).

An analysis was initiated of the reflection of the teacher from the area of natural sciences and strategies were searched for the improvement of the reading comprehension and that promoted the formation of scientific competences implementing the routines of thought, the direct observation of what the context offers, The weekly study sessions of particular context problems (changes in climate, temperature) and the appropriate use of ICT in the classroom. After the implementation of strategies, reading comprehension and the development of scientific thinking were improved, allowing concepts to be constructed, modified and interpreted from the context.

KEY WORDS: Scientific competences, development of scientific thinking, understanding of scientific texts, teaching strategies, TIC.

\section{INTRODUCCIÓN}

La Reflexión Docente, es una estrategia que nos permite analizar y comprender, una situación referente al proceso de enseñanza y aprendizaje, este análisis, intencional o involuntario, revela la transcendencia de la aplicación de determinadas prácticas pedagógicas dentro y fuera del aula. Dicha actividad reflexiva sobre la práctica docente invita a pensar en las acciones educadoras que se dan en los distintos contextos de aprendizaje, con el fin de buscar estrategias de solución a todas aquellas situaciones problemáticas que se van originando en el aula. Tal como afirma Gardner (2005) "los jóvenes deben aprender a pensar de una manera científica si quieren entender el mundo moderno y participar en él".

Este escrito muestra la caracterización de la comprensión lectora en textos científicos en estudiantes de $4^{\circ}$ grado de la Institución Educativa Anaurio Manjarrez La Jagua del PilarLa Guajira. A partir de esto implicará una transformación en la práctica pedagógica en el área de Ciencias Naturales y Educación Ambiental que se irá perfeccionando en el aula, y a la adquisición de saberes pedagógicos.

Una observación hacia problemáticas reflejadas en el aula de clase de $4^{\circ}$ grado de primaria en el área Ciencias naturales y Educación Ambiental de la Institución Educativa Anaurio Manjarrez La Jagua del Pilar- La Guajira se constituye en la herramienta de apertura frente al ejercicio de la investigación, la puerta de entrada de este proceso de búsqueda de explicaciones, de causas y razones, de interrogantes nuevos. Si bien, se ha considerado, en algunos casos y de manera deliberada a la observación como un ejercicio simple, que se basa en instintos y reflejos, se deben tener en cuenta que su naturaleza deriva de los elementos que parten de la intensión, el conocimiento, la 


\title{
Bio - grafía. Escritos sobre la Biología y su Enseñanza. ISSN 2027-1034
}

Edición Extraordinaria. p.p. 1185 - 1195

\author{
Memorias del IX Encuentro Nacional de Experiencias en Enseñanza de la Biología y la \\ Educación Ambiental. IV Congreso Nacional de Investigación en Enseñanza de la \\ Biología.
}

selección y la interpretación. Las Ciencias Naturales en primaria especialmente deben priorizar en hacer preguntas y encontrar caminos, para respuestas con actividades prácticas y aprovechar esa curiosidad innata que tienen los niños y las ganas de explorar el mundo que los rodea.

En este sentido y de acuerdo con Bunge (2000) "la observación se caracteriza como una percepción intencionada e ilustrada porque se hace con un objeto determinado, ilustrada porque va guiada de algún modo por un cuerpo de conocimiento... la observación es selectiva e interpretativa, selectiva porque tiene una finalidad, e interpretativa porque busca entender y explicar lo que se ha observado".

\section{Contexto}

La Institución Educativa Anaurio Manjarrez, de carácter oficial está ubicada en la zona urbana del municipio de La Jagua del Pilar del departamento de La Guajira. Funciona desde el 30 de marzo del 1.992, cuyo único corregimiento es El Plan, es la única Institución Educativa que tiene el municipio, ofreciéndole a la Comunidad Jagüera la modalidad Académica con proyección a la Media Técnica, y con un énfasis en agroindustria. Actualmente la Institución cuenta con 23 Aulas en total, las cuales están distribuidas en dos sedes, alberga 530 estudiantes, distribuidos en los niveles de preescolar, básica primaria y secundaria, en su mayoría provenientes de los estratos 1 y 2 del área urbana y rural, de igual forma consta de 23 docentes y 6 funcionarios administrativos.

La población institucional es muy alegre, bullanguera, humilde, la base de su economía es la agricultura, y los que no poseen tierras propias trabajan por jornadas en el campo. El trabajo se realizó en el aula de clase de 4ำ de primaria con 30 estudiantes: 18 niñas y 12 niños donde la mayoría presenta un tipo de dificultad familiar, unos sin padres dejados a que los abuelos los eduquen, sin mamá por fallecimiento, a veces sin desayuno, padres encarcelados, alcohólicos y donde las problemáticas de comprensión lectora primero se tomaban desde la parte del área lenguaje presentando preocupación en los textos narrativos.

\section{La problemática}

Buitrago (2017) plantea que para evidenciar el pensamiento científico en los estudiantes hay que acabar con los paradigmas, llevando a pensar que el pensamiento científico nace desde nosotros con ese conocimiento pedagógico y disciplinar del área que desempeñemos para que se convierta en nuestra mejor estrategia de formación académica y personal.

La selectividad y la interpretación que se dan a las situaciones encontradas en el aula coloca un reto muy interesante para mejorar las prácticas pedagógicas del salón de clase y a encontrar soluciones al problema más notorio que es la interpretación de textos, ya que a los estudiantes les cuesta expresar pensamiento de acontecimientos cotidianos o textos narrativos (cuento, fabulas, mitos...etc.) y sacar una opinión personal de lo que leyeron. Para esta investigación se les pidió a 30 estudiantes de $4^{\circ}$ que hicieran el ejercicio de comprensión lectora del siguiente texto (Ver imagen 1): 
Bio - grafía. Escritos sobre la Biología y su Enseñanza. ISSN 2027-1034

Edición Extraordinaria. p.p. 1185 - 1195

Memorias del IX Encuentro Nacional de Experiencias en Enseñanza de la Biología y la

Educación Ambiental. IV Congreso Nacional de Investigación en Enseñanza de la Biología.

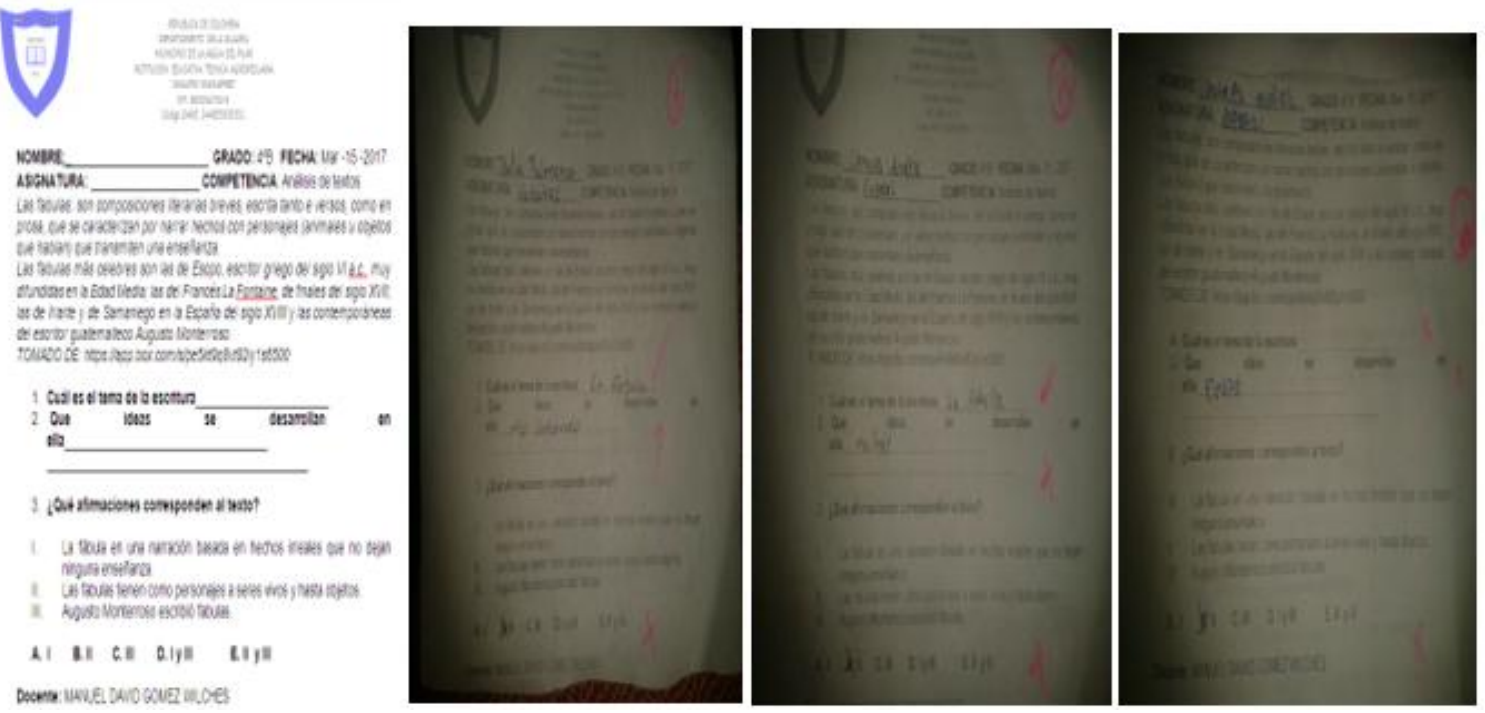

Imagen 1. Prueba de análisis de texto para alumnos de 4ํ I.E. Anaurio Manjarrez Tomada: https://app.box.com/s/pe5kt9q8vt92iy1s6500

A partir de la variedad de textos y las prácticas que se realizan en los colegios parece que el texto narrativo es el que tienen mayor acceso los estudiantes ya que como afirma Colomer y Camps (2000) "el texto narrativo, es, con toda probabilidad, el que más tempranamente se conoce en nuestra cultura [....] se ha destacado la precocidad demostrada por los niños y niñas en el conocimiento de esta macroestructura" (p. 61). Esto propone y a la vez reafirma que los textos narrativos juegan un papel importante en la etapa escolar, concibiéndolos necesarios en la formación lectora de los estudiantes, al igual que son propicios para el mejoramiento de la comprensión de lectura. Sáenz C, Pérez J. (2016). Desde el área de ciencias naturales una de las necesidades primordiales de nuestros educandos es que se apropien de las competencias científicas, pero generando mayor compromiso crítico, aplicando estrategias más llamativas para combatir la dificultad en los procesos de lectura y escritura en los textos narrativos y científicos.

Es bien sabido que la interpretación de texto se da en todas las áreas del conocimiento ciencias sociales, ciencias naturales, lenguaje, matemáticas... etc. Es parte fundamental de las Pruebas Saber que responden los estudiantes de 3ํ. 5, $7^{\circ}$, 9o y $11^{\circ}$ reflejándose más adelante en los resultados académicos, haciendo un rastreo de cómo están los estudiantes, se tomó como referencia los resultados Pruebas Saber año 2015 y 2016 reflejaron los siguientes resultados en materia de competencias lectora (Ver gráfica 1). 
Bio - grafía. Escritos sobre la Biología y su Enseñanza. ISSN 2027-1034

Edición Extraordinaria. p.p. 1185 - 1195

Memorias del IX Encuentro Nacional de Experiencias en Enseñanza de la Biología y la

Educación Ambiental. IV Congreso Nacional de Investigación en Enseñanza de la Biología.
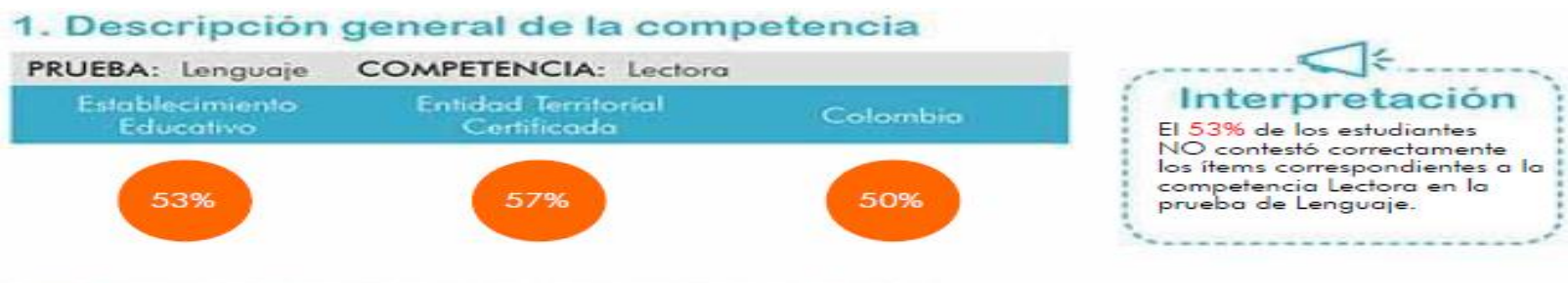

2. Descripción general de los aprendizajes
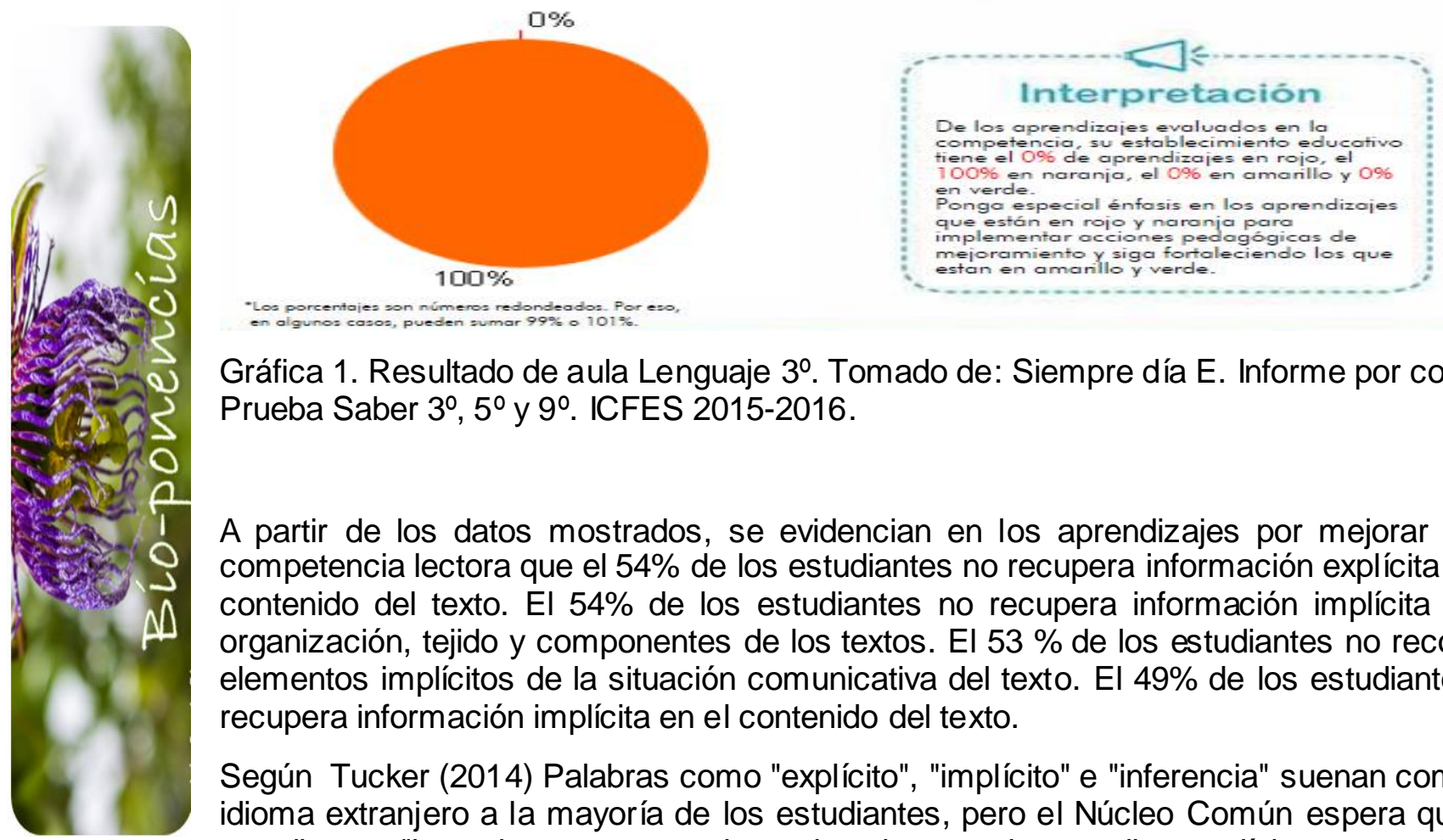

Gráfica 1. Resultado de aula Lenguaje 3‥ Tomado de: Siempre día E. Informe por colegio. Prueba Saber 3ำ 5ำ y 9․ ICFES 2015-2016.

A partir de los datos mostrados, se evidencian en los aprendizajes por mejorar en la competencia lectora que el $54 \%$ de los estudiantes no recupera información explícita en el contenido del texto. El $54 \%$ de los estudiantes no recupera información implícita de la organización, tejido y componentes de los textos. El $53 \%$ de los estudiantes no reconoce elementos implícitos de la situación comunicativa del texto. El $49 \%$ de los estudiantes no recupera información implícita en el contenido del texto.

Según Tucker (2014) Palabras como "explícito", "implícito" e "inferencia" suenan como un idioma extranjero a la mayoría de los estudiantes, pero el Núcleo Común espera que los estudiantes "lean de cerca para determinar lo que el texto dice explícitamente y hacer inferencias lógicas de él". Deben ser capaces de identificar tanto información explícita como implícita, para que puedan hacer inferencias sobre lo que leen.

La escuela está perdiendo su esencia, porque descontextualiza lo que está haciendo y eso genera que las competencias científicas transmitidas no tengan los mejores resultados en el contexto donde se encuentra. Para el caso, el siguiente ejemplo en la Jagua del Pilar - La Guajira: si un docente sabe que la producción de melón, la yuca y el maíz es lo fuerte en la agricultura del pueblo cómo es posible, que de orientaciones de cómo sembrar papa, uvas y otra serie de cosas que el contexto no maneja, aquí juega el conocimiento profesional del profesor de ciencia (CPPC) y sus componentes contextual y disciplinar que promueve el uso de los recursos que el contexto genera. De acuerdo con lo anterior, se debe llegar a un modelo de enseñanza contextualizada que brinde oportunidades justas en aprendizaje y pensamiento para el desarrollo de competencias científicas en la escuela. 
Bio - grafía. Escritos sobre la Biología y su Enseñanza. ISSN 2027-1034

Edición Extraordinaria. p.p. 1185 - 1195

Memorias del IX Encuentro Nacional de Experiencias en Enseñanza de la Biología y la

Educación Ambiental. IV Congreso Nacional de Investigación en Enseñanza de la Biología.

\section{METODOLOGÍA}

Esta investigación se desarrolla desde un enfoque cualitativo de nivel descriptivo, ya que pretende inicialmente fundamentar la contextualización de estrategia pedagógica y caracterizar el proceso de desarrollo para la compresión lectora en la clase de Ciencias Naturales y Educación Ambiental con los estudiantes del grado cuarto de la Institución Educativa Anaurio Manjarrez de la Jagua del Pilar- La Guajira, para realizar la intervención pedagógica y evaluar el proceso.

Se utilizaron modelos de preguntas de comprensión lectora tanto en Lenguaje como en Ciencias Naturales para dar validez a que nuestros estudiantes tienen niveles bajos de comprensión de textos literarios como científicos.

Con un diseño Investigación - acción como lo afirma Kemmis (2002) que es una forma de indagación autorreflexiva que emprenden los participantes en situaciones sociales en orden a mejorar la racionalidad y la justicia de sus propias prácticas, su entendimiento de las mismas y las situaciones dentro de las cuales ellas tienen lugar (Kemmis en Williamson, 2002).

\section{RESULT ADOS Y DISCUSIÓN}

Haciendo un análisis se parte de la reflexión del docente desde el área de Ciencias Naturales se buscan estrategias para la mejora de la comprensión lectora y que promuevan la formación de competencias científicas aplicando: rutinas de pensamiento, observación directa de lo que nos ofrece el contexto en materia de ciencias, sesiones de estudio semanal de problemas particulares del contexto (cambios de clima, temperatura,...) y uso adecuado de las TIC en el aula. 
Bio - grafía. Escritos sobre la Biología y su Enseñanza. ISSN 2027-1034

Edición Extraordinaria. p.p. 1185 - 1195

Memorias del IX Encuentro Nacional de Experiencias en Enseñanza de la Biología y la

Educación Ambiental. IV Congreso Nacional de Investigación en Enseñanza de la Biología.

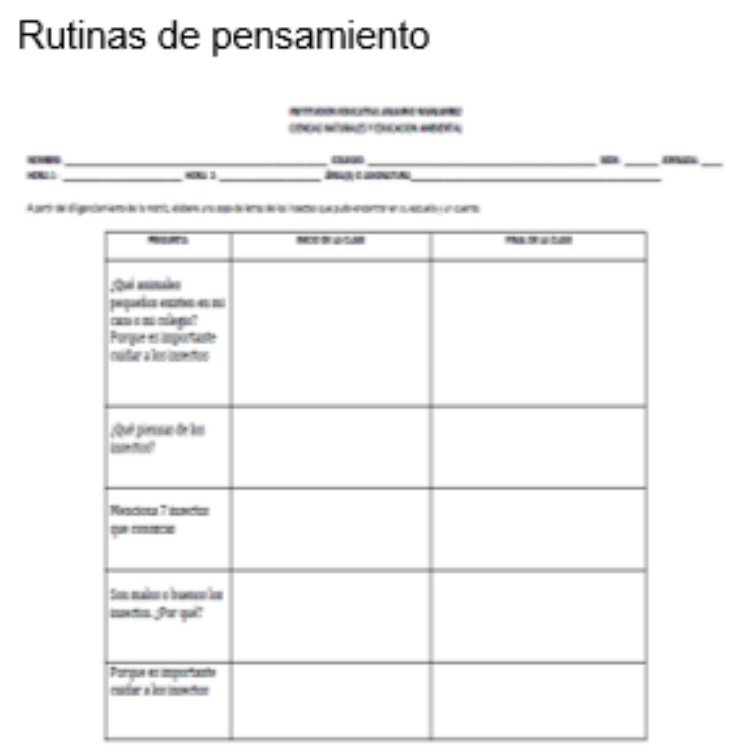

Observación directa

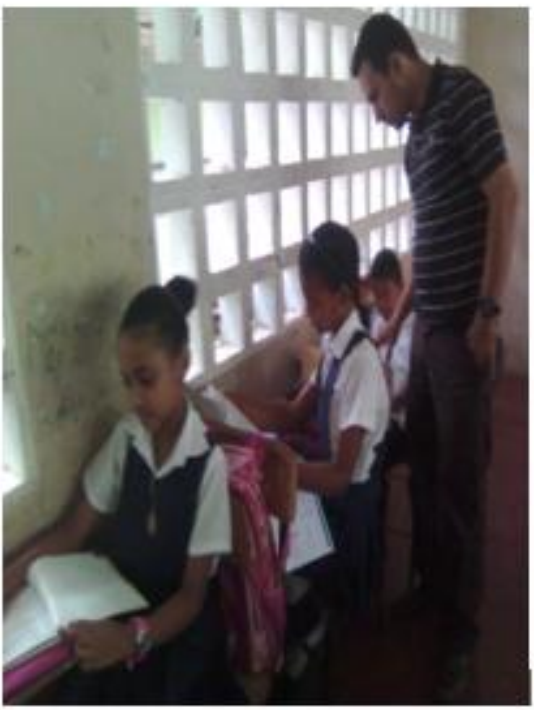

Uso de las TIC

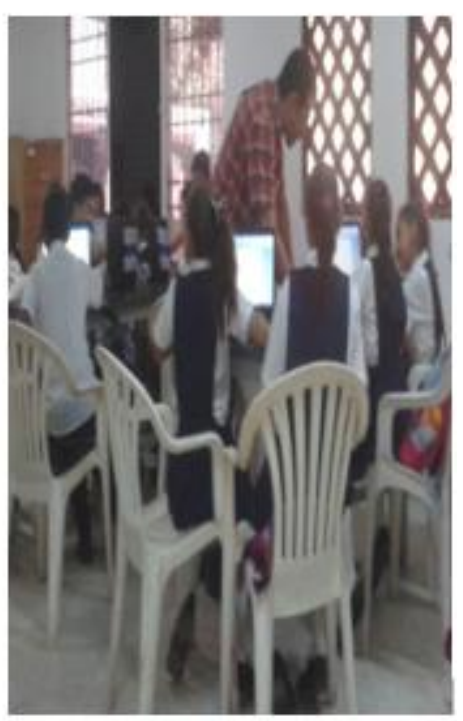


Bio - grafía. Escritos sobre la Biología y su Enseñanza. ISSN 2027-1034

Edición Extraordinaria. p.p. 1185 - 1195

Memorias del IX Encuentro Nacional de Experiencias en Enseñanza de la Biología y la

Educación Ambiental. IV Congreso Nacional de Investigación en Enseñanza de la Biología.

Versos Vallenatos
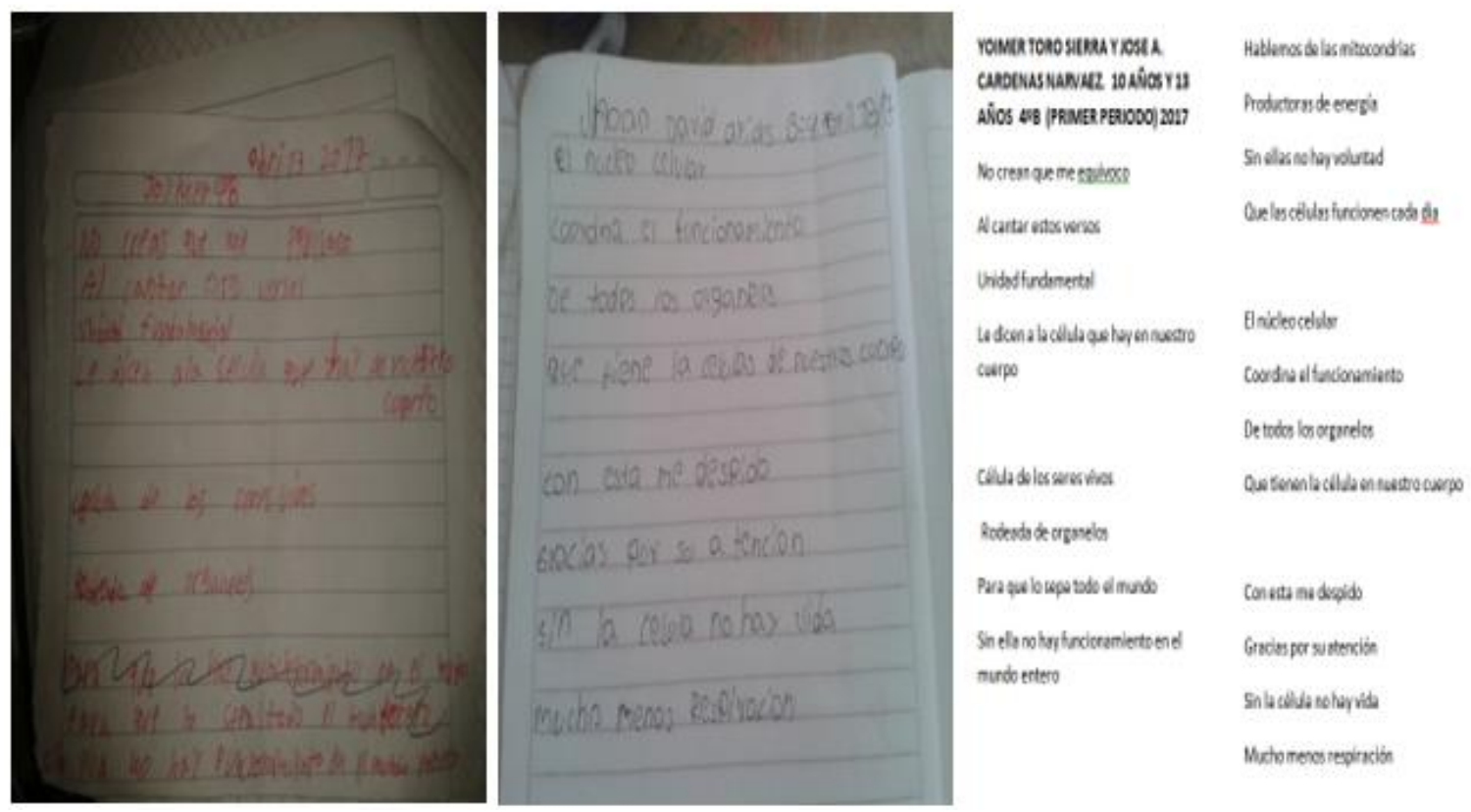

Imagen 2. Estrategias utilizadas para mejorar la comprensión de textos científicos y el desarrollo de competencias científicas.

Marqués (2004) citado por Carneiro R. (2009) menciona que las TIC como instrumentos configuradores de entornos o espacios de trabajo y de aprendizaje. En síntesis, se espera que el docente del siglo XXI sea altamente competente en el diseño de entornos de aprendizaje donde se utilicen de manera profusa y pertinente los medios de comunicación y las TIC, aprovechando todo su potencial informativo, comunicativo y motivador

Con la utilización de las TIC en Ciencias Naturales, permite que los estudiantes se incluyan y pertenezcan a un mundo global que evoluciona vertiginosamente; que se da en el aprendizaje a través de la información numerosa y variada utilizando buscadores, bibliotecas virtuales y otra base de datos que le permita mejorar esa comprensión lectora en el área de Ciencias Naturales y hacer más atractivo el proceso de enseñanza y aprendizaje. En esa línea, Barreto (2017) plantea que las necesidades educativas de La Guajira requieren cada vez de la cualificación de profesionales idóneos capaces de innovar y pretender los avances y requerimientos que se dan en el mundo de hoy, que permitan a nuestros estudiantes no solo llenarlos de conocimientos teóricos, sino todo un abanico de posibilidades y oportunidades que puedan poner en práctica en sus escenarios de desarrollo. 


\title{
Bio - grafía. Escritos sobre la Biología y su Enseñanza. ISSN 2027-1034
}

\section{Edición Extraordinaria. p.p. 1185 - 1195}

\author{
Memorias del IX Encuentro Nacional de Experiencias en Enseñanza de la Biología y la \\ Educación Ambiental. IV Congreso Nacional de Investigación en Enseñanza de la \\ Biología.
}

El conocimiento profesional del profesor de ciencias (CPPC) es la herramienta fundamental de nuestro quehacer ya que permite el desarrollo de los contenidos en el aula de clase por lo tanto debe ir ligado al conocimiento pedagógico del contexto en el cual se va a enseñar. Para conocer una disciplina lo fundamental es tener claro lo conceptos estructurantes de ella y saber cómo se ha ido construyendo esa disciplina, eso nos permite como profesores tener mayores oportunidades de aprender y de articularlos a otros componentes del CPPC.

Siendo mejores profesores promovemos principios de equidad, justicia y una calidad de educación para Colombia. Para promover transformaciones educativas en el aula el proceso de investigación - acción pedagógica, que viene siendo aquella que el profesor es capaz de hacer con su reflexión permanente en el salón de clase y de este análisis es que surgen los diferentes problemas que encontramos en el desarrollo de la clase para evidenciarlos y darles las mejores soluciones posibles con estrategias pedagógicas que genera ese cambio en el alumnado. Cuando hablo de ese ciclo de investigación - acción que parte de un problema que observo, realizo una reflexión, planteo estrategias y las ejecuto, con esto promuevo una intensión de cambio.

\section{CONCLUSIONES}

Para concluir como profesor de Ciencias Naturales se tiene la obligación de realizar juicios críticos de la práctica pedagógica y la participación de un trabajo en equipo constante; si bien la cultura individualista domina hoy en día, el trabajo en equipo es necesario, por no decir fundamental, para lograr buenos resultados en cuanto al aprendizaje. Estas evidencias o registros podemos hacerlos en diarios de campo como ayudas pedagógicas para una sistematización, esto permite analizar problemas comunes aunando criterios de acciones coherentes e inmediatas, que buscan soluciones desde el conocimiento práctico y teórico, en una organización educativa basada en la cooperación y la colaboración (Fuentes X. 2011 p. 4).

Sabemos que la investigación pedagógica busca mejorar el aprendizaje, despertar la curiosidad del docente y llevar la investigación a la acción, como docente estamos en la obligación de investigar más en el aula, ser un profesional reflexivo y mejorar la práctica educativa.

Conocer las condiciones de vida de cada lugar es importante para poder adecuar la práctica tomando en cuenta las necesidades de cada niño que se desenvuelve en el aula y la naturaleza de cada una de las variables y de las interacciones que se dan en el contexto nos indicarán hasta qué punto nuestros propósitos educativos pueden ser alcanzables. Los distintos contextos de trabajo, permiten estar al tanto de las características y del entorno en que se encuentra la escuela donde llevaremos a cabo nuestras prácticas. 
Bio - grafía. Escritos sobre la Biología y su Enseñanza. ISSN 2027-1034

Edición Extraordinaria. p.p. 1185 - 1195

Memorias del IX Encuentro Nacional de Experiencias en Enseñanza de la Biología y la Educación Ambiental. IV Congreso Nacional de Investigación en Enseñanza de la Biología.

Gracias a la observación directa, rutinas de pensamiento, la creación de versos vallenatos para retener conceptos propios en Ciencias Naturales y educación ambiental y el uso adecuado de las TIC en el aula clase se pudo mejorar la comprensión lectora y del desarrollo del pensamiento científico para permitir construir conceptos, modificarlos y descubrir interpretaciones desde el contexto propio de La Guajira.

\section{REFERENCIAS BIBLIOGRÁFICAS}

$\checkmark$ BARRETO C. (2017). Conferencia inaugural. Primer Coloquio Departamental de Desarrollo del Pensamiento Científico y Tecnológico. La Guajira. Universidad de La Sabana. Maestría en Pedagogía. Ext. La Guajira.

$\checkmark$ BUITRAGO J. (2017). Conferencia inaugural. Primer Coloquio Departamental de Desarrollo del Pensamiento Científico y Tecnológico. La Guajira. Universidad de La Sabana. Maestría en Pedagogía. Ext. La Guajira.

$\checkmark$ BUNGE, M. (2000). La investigación científica. Barcelona: Ariel. Recuperado de: https://guiadetesis.files.wordpress.com/2012/07/bunge_mario_la_investigacion_cientifica.pdf

$\checkmark$ CARNEIRO R., TOSCANO J., DÍAZ T. (2009). Los desafíos de las TIC para el cambio educativo. Fundación Santillana. España.

$\checkmark$ COLOMER, T \& CAMPS A. (2002). Enseñar a leer, enseñar a comprender. Madrid: Celeste Ediciones/M.E.C

$\checkmark$ FUENTES X. (2011). Reflexión en torno a la gestión de aula y a la mejora en los procesos de enseñanza y aprendizajes. Revista lberoamericana de Educación. pág. 4.4 Recuperado https://www.google.com.co/url?sa=t\&rct=j\&q=\&esrc=s\&source=web\&cd=2\&cad=rja \&uact $=8$ \&ved=0ahUKEwihxJrm2K7SAhUCSyYKHfkYAl8QFgggMAE\&url=http\%3A \%2F\%2Frieoei.org\%2Fjano\%2F4048Villalobos_Jano.pdf\&usg=AFQjCNF_98NDm OTxII7Tmmg3lya86DJpJg\&sig2=3 lipPp814mqwnHfRSbgX5g 
Bio - grafía. Escritos sobre la Biología y su Enseñanza. ISSN 2027-1034

Edición Extraordinaria. p.p. 1185 - 1195

Memorias del IX Encuentro Nacional de Experiencias en Enseñanza de la Biología y la

Educación Ambiental. IV Congreso Nacional de Investigación en Enseñanza de la Biología.

$\checkmark \quad$ ICFES. (2015). Resultado de aula Lenguaje $3^{\circ}$. Siempre día E. Informe por colegio.

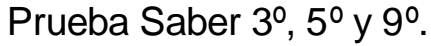

$\checkmark$ SÁENZ C, PÉREZ J (2016) Incidencia de una Propuesta Pedagógica para Mejorar la Comprensión de Lectura en los Estudiantes del Grado Cuarto del Colegio Tibabuyes Universal. [Tesis de Maestría en Pedagogía]. Universidad de la Sabana. 2016. pág. 23.)

$\checkmark$ TUCKER C. (2014). Common Core: Explicit vs. Implicit Information. [Mensaje en un blog]. Recuperado de http://catlintucker.com/2014/08/common-core-explicit-vsimplicit-information/

$\checkmark$ VARGAS B. (2016) De la observación a la descripción. UN DESAFIO POSIBLE. Revista Magisterio. Vol. 82. Pág. 29.

$\checkmark$ WILLIAMSON, G. (2002) Investigación Acción Participativa Intercultural en Comunidades Educacionales y Locales. Universidad de La Frontera, Proyecto Kelluwün, Temuco. 